\title{
Crack initiation and propagation mechanisms of an extruded dual-phase Magnesium-Lithium alloy in very high cycle fatigue regime
}

\author{
Xiangyu WANG ${ }^{1}$, Chao HE ${ }^{1}$, Xue $\mathrm{LI}^{1}$, YongJie Liu ${ }^{1}$, Qingyuan Wang ${ }^{1}$, Hong ZHANG ${ }^{1}$, \\ Lang LI ${ }^{1}$, and Chong Wang ${ }^{1}$ \\ ${ }^{1}$ Sichuan University
}

April 27, 2021

\begin{abstract}
Ultra-light $\mathrm{Mg}-\mathrm{Li}$ alloy is a promising alloy in aerospace since it is known to the lightest structural alloy at present, but its fatigue behaviors remain to be explored. This work focuses on very-high cycle fatigue (VHCF) strength and small crack initiation behaviors of an extruded dual-phase Mg-Li alloy (LZ91). The fatigue strength of the LZ91 alloy at 109 cycles is about $78 \mathrm{MPa}$, and the fatigue ratio is approximately 0.46. Microstructure characterization demonstrates that fatigue crack tends to initiate from the $\beta$-Li phase-enriched region. The $\alpha-\mathrm{Mg}$ phase, presenting $<10-10>$ fiber texture with the basal plane, deforms hardly along the extrusion direction and acts as an enhanced phase compared with the $\beta$-Li phase. The deformation discrepancy localizes cyclic plasticity at the Li phase and finally leads to the fatigue crack initiation.
\end{abstract}

Crack initiation and propagation mechanisms of an extruded dual-phase Magnesium-Lithium alloy in very high cycle fatigue regime

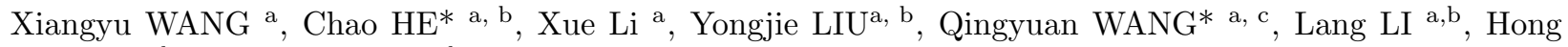
ZHANG $^{\mathrm{a}, \mathrm{b}}$, Chong WANG ${ }^{\mathrm{a}, \mathrm{b}}$

a Key Laboratory of Deep Earth Science and Engineering, Ministry of Education, College of Architecture and Environment, Sichuan University, Chengdu 610065, China

b Failure Mechanics \& Engineering Disaster Prevention and Mitigation, Key Laboratory of Sichuan Province, Sichuan University, Chengdu 610065, China

c School of Architecture and Civil Engineering, Chengdu University, Chengdu 610106, China

Corresponding authors:

Dr. Chao HE

Sichuan University

Email: hechao@scu.edu.cn

Prof. Qingyuan WANG

Sichuan University

Email: wangqy@scu.edu.cn

Abstract: Ultra-light Mg-Li alloy is a promising alloy in aerospace since it is known to the lightest structural alloy at present, but its fatigue behaviors remain to be explored. This work focuses on very-high cycle fatigue 
(VHCF) strength and small crack initiation behaviors of an extruded dual-phase Mg-Li alloy (LZ91). The fatigue strength of the LZ91 alloy at $10^{9}$ cycles is about $78 \mathrm{MPa}$, and the fatigue ratio is approximately 0.46 . Microstructure characterization demonstrates that fatigue crack tends to initiate from the $\beta$-Li phaseenriched region. The $\alpha$-Mg phase, presenting $<10-10>$ fiber texture with the basal plane, deforms hardly along the extrusion direction and acts as an enhanced phase compared with the $\beta$-Li phase. The deformation discrepancy localizes cyclic plasticity at the Li phase and finally leads to the fatigue crack initiation.

Keywords:Magnesium-lithium alloy; very-high cycle fatigue; crack initiation and propagation; microstructural sensitivity.

\section{Introduction}

In recent decades, magnesium alloy is one of the lightest structural metallic materials, the advantages of this alloy are its outstanding specific strength, sound damping capabilities [1-3]. However, due to its hexagonal closed-packed (HCP) lattice structure, magnesium alloy possesses limited slip systems, low ductility, and unfavorable formability at room temperature [4-6]. Recent studies have shown that the formability and ductility of magnesium alloys would be highly improved by adding an appropriate amount of Lithium (BCC, body-centered cubic structure) [7,8]. It is reported that Lithium addition would significantly decrease the CRSS value of non-basal slip systems by introducing more slip systems and reducing the c/a ratio [9]. Besides, with the addition of lithium, the lightest metal (about $0.534 \mathrm{~g} / \mathrm{cm}^{3}$ ), the $\mathrm{Mg}$ - $\mathrm{Li}$ dual-phase alloy could significantly reduce its density without losing too much mechanical strength [10, 11]. Therefore, $\mathrm{Mg}$ $\mathrm{Li}$ alloy is widely considered as an ideal structural material in the aerospace, automotive, and electronic industry. Usually, the structural material is subjected to repeated fatigue loadings during its service life, which may exceed more than $10^{7}$ cycles and reach the VHCF (very-high cycle fatigue) regime [12-14]. However, knowledge of the VHCF properties of Mg-Li is fairly limited, which prevents its wide application. Thus, it is essential to fully investigate its VHCF behavior.

As we all know, small crack initiation behavior accounts for the majority (about 90\%-95\%) of the total fatigue life [15-17] of the material and it is closely related to the microstructure of the material. For conventional magnesium alloy with a single phase of HCP structure, it is widely reported that crack initiation is closely related to the deformation texture. Crack is mainly induced by twinning deformation in $\mathrm{Mg}$ alloys with strong deformation texture [18], while basal slip-induced crack is the predominant crack initiation mode in $\mathrm{Mg}$ alloys with weak deformation texture [19-21]. In the case of dual-phase $\mathrm{Mg}$-Li alloy, the plastic deformation behavior of the material becomes more complicated with the introduction of the $\beta$-Li phase. Currently, plastic deformation and fracture behavior have been examined by some researchers [22, 23]. For instance, Guo et al. [23] investigated the slip behavior of dual-phase $\mathrm{Mg}$-Li alloy by tracing the microstructure and texture evolution during the rolling procedure. It proposed that $\{001\}<110>$ was the dominant texture component in the $\beta$-Li phase and basal slip, pyramidal I and II slip worked together to coordinate deformation strain in the $\alpha$-Mg phase. On the other hand, Dong et al. [22] found that deformation and fracture occurred mainly in the $\beta$-Li phase, and $\alpha-\mathrm{Mg}$ phase participated in fracture at high tensile speed. However, what remains unclear is how the $\alpha-\mathrm{Mg}$ and $\beta-\mathrm{Li}$ phase coordinate the deformation strain as well as the fracture mechanism of dual-phase $\mathrm{Mg}$ - Li alloys in the regimes of high-cycle and very high-cycle fatigue.

Therefore, the purpose of this research has been to study the behavior of dual-phase Mg-Li alloy LZ91 in the VHCF regime. The relation between fatigue crack initiation and propagation and the microstructure of the material was carefully investigated through microstructural characterization and quantitative statistics. This research may increase understanding of the formation mechanism of the fatigue crack of LZ91 alloy and potentially provide a way to improve its fatigue properties.

\section{Material and experimental procedure}

\subsection{Materials}

The material utilized in the present work is an as-extruded dual-phase magnesium-lithium alloy LZ91. The material was prepared by melting high purity element magnesium, lithium, and zinc in a vacuum induction 
melting furnace under a protective gas of high purity argon gas, followed by stirring and a setting of 3 min, then they were poured into the cylindrical billets with a temperature of 730 . Then it was held at 250 for 1 $\mathrm{h}$ and to obtain an as-cast ingot. After that, the obtained as-cast ingot was extruded at the temperature of 200 with an extruding speed of $2 \mathrm{~m} / \mathrm{min}$. Finally, an extruded rod with dimensions of $\$ 110 \mathrm{~mm} \times 400 \mathrm{~mm}$ was obtained. The chemical compositions of the LZ91 alloy are displayed in Table $\mathbf{1}$, and the mechanical properties are listed in Table 2 , respectively.

In order to observe the microstructure of the LZ91 alloy, the specimen surface was ground with 800, 1200, 2000 grits silicon carbide paper, respectively, then it was an electrolytic etched perchloric acid-ethanol solution (perchlorate acid: ethanol =1: 9) for 90 seconds. The metallography of the cross-section and side surface of the specimen was presented in Figure 1, which was characterized by an optical microscope (OM, OLYMPUS GX53). It can be seen clearly that the microstructure of LZ91 alloy mainly consists of a brighter $\alpha$-Mg phase and a relatively darker $\beta$-Li phase. Moreover, it should be noted that there is a significant difference between the microstructure of the cross-section and the side surface of the specimen. The $\alpha$-Mg phase inFigure 1a distributes in $\beta$-Li phases with irregular shapes, while Figure 1b shows that the $\alpha$-Mg and $\beta$-Li phases were both elongated along axial direction due to the radial extrusion.

\subsection{Experimental procedure}

\subsubsection{Nano-indentation}

Nano-indentation was applied to characterize the hardness values of the $\alpha$-Mg phase and $\beta$-Li phase. The nano-indentation test sample was cut by a precision cutting machine, then it was mechanically ground. After that, the electrolytic etching was employed to present the microstructure and distinguish the $\alpha-\mathrm{Mg}$ phase and $\beta$-Li phase of the material, and eliminate the residual stress as well. The nano-indentation tests were performed in KLA iNano, the maximum load and target depth were set as $20000 \mu \mathrm{N}$ and $1000 \mathrm{~nm}$ to make indentations inner $\alpha$-Mg phase and $\beta$-Li phase.

\subsubsection{Fatigue testing}

Data on long-life fatigue fracture of LZ91 were obtained by the fatigue tests, which were conducted on an ultrasonic fatigue test system (Shimadzu USF-2000) at room temperature in the air. The resonance interval was $100 \mathrm{~ms}$ per $300 \mathrm{~ms}$, and the stress ratio $\mathrm{R}=-1$, up to a limit of $10^{9}$ cycles except for failure. Fatigue test specimens were performed on hourglass-shaped specimens, and the length of the specimen was designed to give it a resonance of $20 \mathrm{kHz}$. The detailed specimen configurations is shown in Figure $\mathbf{3}$. Before fatigue testing, surfaces of specimens were polished in advance by different grades of abrasive papers and cloths to eliminate the effect of surface roughness on fatigue behavior. Temperature increment caused by cyclic loadings used in this work was measured to be no more than 5 during fatigue tests by an infrared camera (NEC-Avio R500EX). Besides, compressed cold air was adopted to cool the specimens during fatigue tests.

\subsubsection{Microstructure observation methods}

After fatigue tests, the microstructure of the material was characterized by a scanning electron microscope (SEM, JEOL 7800F). The detection mode was SEI (secondary electronic imaging) mode, and the acceleration voltage is set to $15 \mathrm{kV}$, respectively. The SEM observation area was concentrated on the fracture surface and specimen surface. Especially, we carried out higher magnifications for the regions near the crack initiation sites, which is focused on the crack initiation behavior of the material. On the other hand, the EBSD (electron backscatter diffraction) technique was applied to determine the grain orientation of the material. The area detected is the cross-section of the material, and the diffraction direction is perpendicular to the cross-section.

\section{Experimental results}

\subsection{Texture characterization}

The microstructure of the material was characterized by the EBSD technique before fatigue tests. Figure 2 displays the EBSD information of the cross-section of the material, including the grain orientation map, 
the pole figure, and the inverse pole figure. The striped phase in the grain orientation map in different colors is the $\alpha$-Mg phase. It should be noted that the $\beta$-Li phase can not be resolved due to its high metal activity and high oxidation, thus it acts as a black matrix in the grain orientation map. The EBSD results, especially in the pole figure, show that the $\alpha-\mathrm{Mg}$ phase exhibits a strong texture along the axial direction of the material, the grain orientation of most grains is around $\{10-10\}$ and $\{11-20\}$ (the cylindrical directions of the hexagonal close-packed structure). In other words, the basal plane of most grains is parallel or nearly parallel to the axial direction of the material. It is typical for the magnesium and its alloys to exhibit a texture after extrusion, which mainly results from recrystallization and a unidirectional deformation during extrusion [24-26].

\subsection{Nano-indentation results}

The mechanical properties of $\alpha-\mathrm{Mg}$ and $\beta$-Li phase obtained by the nano-indentation test are listed in Table $\mathbf{3}$, and the loading-displacement curves are shown in Figure $\mathbf{4}$, respectively. By comparing the mechanical properties of these two phases, it can be found that the hardness and elastic modulus values of the $\alpha-\mathrm{Mg}$ phase and the $\beta$-Li phase are fairly close, about $75 \mathrm{HV}$ and $50.5 \mathrm{GPa}$, respectively. Notably, however, the loading-depth curve of the $\alpha-\mathrm{Mg}$ phase varies greatly from the one of $\beta$-Li phase. It can be seen that the depth of the indentation in the $\beta$-Li phase increased smoothly with the increasing of the loading stress applied by the probe. As shown inFigure 4a, however, the loading-depth curve of the $\alpha$-Mg phase is quite discontinuous and presents a ladder-like pattern. Similar results were obtained by some previous nanoindentation experiments of Mg-Li alloy done by V. Kumar et al. and W. Wang et al. [27-29], and this phenomenon was named as "pop-in" effect. Guo et al. [30] carried out nano-indentation teste on magnesium grains of $\{10-10\}$ and $\{11-20\}$ crystal planes. It was concluded that the "pop-in" effect in loading-depth curves is ascribed to the initiation of $\{10-12\}$ extension twinning. This can be indirectly supported by the EBSD results of the material. As stated in section 3.1, the axial grain orientation of $\alpha$-Mg grains is mostly concentrated in $\{10-10\}$ and $\{11-20\}$ cylindrical directions. Therefore, when the $\alpha-\mathrm{Mg}$ phase is subjected to an axial loading applied by the probe, the twinning deformation will be activated discontinuously when the shearing stress reaches the critical value, as presented in the schematic diagram in Figure $\mathbf{4} \mathbf{a}_{\mathbf{1}}$. On the other hand, due to the BCC (body-centered cubic) structure, the $\beta$-Li phase possesses 48 slip deformation systems, which is far more than that of the HCP structure. In other words, there are more slip systems for $\beta$-Li grains to coordinate the plastic deformation when they are subjected to axial loadings. For these reasons, the displacement increases more continuously in the loading-displacement curve of the $\beta$-Li phase as compared with that of the $\alpha-\mathrm{Mg}$ phase.

\subsection{Very high-cycle fatigue}

The S-N data associated with stress amplitudes $\sigma_{\mathrm{a}}$ and fatigue life $\mathrm{N}_{\mathrm{f}}$ of LZ91 alloy under asymmetric axial loading with a stress ratio of $\mathrm{R}=-1$ are shown in Figure $\mathbf{5}$. The $\mathrm{S}-\mathrm{N}$ data of some other $\mathrm{Mg}$ - $\mathrm{Li}$ alloys and magnesium alloys were also included for comparison [19, 31, 32]. "Run-out specimens" represents that the fatigue life of the specimen reached a limit of $1 \times 10^{7}$ (for LAZ832-0.5Y alloy) and $1 \times 10^{7}$ (for the rest three alloys). The nonlinear fitting curves of these fatigue data were obtained by Basquin's equation, which were plotted by dash lines.

It can be seen that the S-N curve of LZ91 alloy exhibits a continuous decreasing trend, even in the range of $10^{7}-10^{9}$ cycles. By comparing the S-N curve of LZ91 alloy and LAZ832-0.5Y alloy, it can be found that the curve of LZ91 alloy decreases much more slowly. Meanwhile, the fatigue performance of the LZ91 alloy is much better than that of the LAZ832-0.5Y alloy when the fatigue life exceeds $10^{5}$ cycles. As compared to conventional magnesium alloys [19,32], whose fatigue tests were also conducted at $\mathrm{R}=-1$ and a loading frequency of $\sim 20 \mathrm{kHz}$, the fatigue resistance of the LZ91 alloy is obviously lower, about 20-35 MPa. However, as the fatigue life approaching $10^{9}$ cycles, the fatigue strength gap between them is narrowing, less than $20 \mathrm{MPa}$ at $10^{9}$ cycles. Due to the significant difference in mechanical properties between LZ91 and these two alloys, the lag of LZ91 in fatigue strength seems inevitable. After that, in order to compare the fatigue performance of these alloys, we calculated their fatigue ratios (the ratio of fatigue strength to the ultimate tensile strength), and the results are displayed in Table 4 . Clearly, the fatigue ratio of the LZ91 alloy, about 
0.46 , is significantly higher than that of the other three alloys $(0.29,0.27,0.30$ for LAZ832-0.5Y alloy, ZK60, AZ31, respectively), which indicates that the LZ91 alloy exhibits relatively good fatigue performance.

\subsection{Observation on fracture surfaces}

After ultrasonic fatigue tests, the scanning electron microscope was applied to characterize the fracture morphologies of failed specimens.Figure 6 shows typical SEM images of two representatives failed specimens at different magnifications. It can be clearly seen that fatigue crack nucleated and initiated from the specimen surface and then continue to expand, which resulting in the ultimate fatigue failure, as presented in Figures $\mathbf{6 a}$ and $\mathbf{6 e}$. Meanwhile, the crack growth direction can be determined according to the radial ridge of the material fracture.

Figures $\mathbf{6 b}$ and $\mathbf{6 f}$ display the enlarged images of the regions near the crack initiation sites of the two failed specimens, respectively. Obviously, along the growth direction of the crack, the fracture morphology varies greatly with the crack grows deeper, which is quite analogous to that of high-strength steels and titanium alloys $[17,33,34]$. Firstly, the regions near the crack initiation sites, with a distance of about $100 \mu \mathrm{m}$, present a relatively smooth morphology. As the region went further away from the crack initiation sites, the fracture morphology became rougher than Zone I. Meanwhile, lots of radial ridges can be seen in Zone II. As the distance goes further, no characteristic morphology but radial streaks can be observed in the regions far away from the crack initiation sites. In summary, according to the difference in morphology, we utilized dash lines to divide the fracture surface into three distinct regions: Zone I, Zone II, and Zone III. These regions corresponding to three stages of crack initiation and propagation, Zone (I): crack initiation region; Zone (II): short crack propagation region; (III): long-crack propagation region.

High-magnification images of Zone I and Zone II are presented inFigures $\mathbf{6 c}$ and $\mathbf{6 g}$. It is obvious that there are much more striped crystallographic profiles in Zone II comparing to Zone I. It can be seen more clearly in Figures $\mathbf{6 d}$ and $\mathbf{6 h}$. By comparing the striped profiles with the $\alpha$-Mg grains in the metallographic diagram of the material, it was found that they are highly similar in appearance as well as area proportion. Namely, these striped crystallographic profiles result from the fracture of the $\alpha$-Mg grains near the crack initiation site. It has been reported in some previous researches that there exist strong and weak phases in duplex stainless steel [35-38]. The crack initiation and propagation behaviors are closely related to the coordinated plastic deformation of the two phases $(\gamma$-austenite phase with faced centered cubic structure and $\alpha$-ferrite phase with body centered cubic structure). On one hand, when the hardness of the two phases differs greatly, the crack tends to initiate in the weaker phase of the material. In this case, the phase with much higher hardness is a hard phase in the material. For instance, M.W. Tofique et al [35]. and R. Strubbia et al. [37] found that since the hardness of ferrite grains is much lower than that of austenite grains, the plastic damage accumulation mainly in the ferrite grains. On the other hand, the crack nucleation will be highly affected by the microstructure (grain orientation, grain boundary, etc.) of the material when the hardness of these two phases differs slightly [35, 38]. As a structural alloy with a dual-phase structure, the LZ91 alloy may possess hard and soft phases as well. Therefore, we investigated the microstructure of two phases of the LZ91 alloy in the discussion section and attempted to associate it with crack initiation and propagation.

\subsection{Morphology of specimen surfaces}

Figure 7 presents an oblique view of the specimen surface near the crack initiation site, the $\alpha$-Mg grains were indicated by white arrows. Note that the crack exhibit different characteristics when passing through the $\alpha-\mathrm{Mg}$ phase and $\beta$-Li phase. The fatigue crack passes directly through the $\beta$-Li phase in the process of crack growth. On the contrary, the crack presents a 'zigzag' pattern when passing through the $\alpha$-Mg grains, as enclosed by yellow dash circles. An enlarged optical image of a second crack near the crack initiation site is presented inFigure $\mathbf{8}$. Clearly, there are numerous turning points in the crack path, especially when the crack passes through $\alpha-\mathrm{Mg}$ grains, making the crack appear pretty tortuous. This is quite analogous to the fatigue behavior of duplex stainless steel [38]. It was reported that the fatigue crack growth was affected by the slip directions of the $\gamma$-austenite grains, which is a relatively hard phase in the material. The 
crack path appears quite tortuous results from the influence of the local microstructure. Therefore, it can be inferred that the $\alpha-\mathrm{Mg}$ phase may also act as a hard phase (i.e. strengthening phase) in the material. The microstructure (grain orientation or grain boundary) of the $\alpha$-Mg phase would hinder the initiation and early propagation of fatigue crack to a certain extent. In the following discussion section, we attempt to quantitatively analyze and figure out how the local microstructure has a macro effect on the crack behavior of the material.

\section{Discussion}

As have been highly stressed in extensive previous studies [15-17], small crack initiation and propagation account for the majority of the total fatigue life of metal materials. In other words, crack initiation and early propagation play an essential role in the fatigue behaviors of metal materials. Therefore, we focus on the small crack initiation and propagation behaviors of LZ91 alloy as well as the influencing factors in the following discussion section.

\subsection{Fracture morphology analysis based on fracture mechanics calculation}

To further investigate the nature of fatigue crack initiation and propagation behavior, quantitative fracture analysis was performed with the help of fracture mechanics. The stress intensity factor $\Delta \mathrm{K}$ of the fracture surface of failed specimens was calculated. For surface crack initiation in the present work, Zone I, II can be approximately regarded as a semi-elliptic. The areas of Zone I and Zone II were measured and their square root values $\sqrt{ }$ area, which was obtained by projecting on the plane perpendicular to the loading axial, were used to calculate the $\Delta \mathrm{K}$ values, given by the equation for surface cracks $[16,39]$ :

$$
\Delta K=0.65 \sigma \sqrt{\pi \cdot \sqrt{a r a}}
$$

Where $\sigma$ is the maximum stress amplitude of cyclic stress for $\mathrm{R}=-1$, and 0.65 is the calculated parameter for surface crack initiation.Figure 9 demonstrates the calculated results of $\Delta \mathrm{K}$ of Zone I and Zone II together with the values of the applied stress amplitude and the fatigue life. Obviously, the values of $\Delta \mathrm{K}$ Zone I and $\Delta \mathrm{K}$ Zone II maintain nearly constant levels regardless of the fatigue strength and the fatigue life. The average $\Delta \mathrm{K}$ Zone Ivalue was evaluated to $1.44 \mathrm{MPa} \cdot \mathrm{m}^{1 / 2}$ and the corresponding value of $\Delta \mathrm{K}$ Zone II was a little higher, about $2.40 \mathrm{MPa} \cdot \mathrm{m}^{1 / 2}$, respectively. Compared with some previous investigations[40, 41], the $\Delta \mathrm{K}$ Zone II is slightly lower than that of some conventional magnesium alloys, which is usually considered as a threshold of stable crack propagation. But it is still within the range of the reported crack growth thresholds of magnesium alloys, about 1.2-5.0 MPa ${ }^{1 / 2}$ [40-42]. Therefore, the $\Delta \mathrm{K}$ value of Zone II can be regarded as the crack propagation threshold of the material, which corresponds to the threshold for stable crack propagation.

Therefore, in the present case, the fatigue failure process of the LZ91 alloy can be divided into three main stages according to the differences in the $\Delta \mathrm{K}$ values and the characteristics of the fracture surface. As demonstrated in Section 3.3, the crack initiation region (Zone I) presents a relatively special morphology, which is similar to the rough area (RA) and facet morphology of some structural alloys [43-45]. In the titanium alloys [44], the facets were formed due to the inconsistent plastic strain of the two phases in the crack initiation region. Based on this, it can be preliminarily deduced that due to the difference in the plastic deformability of the two phases in the material, the plastic strain was highly concentrated in one of them. Subsequently, the local deformation inhomogeneity of the two phases would lead to the nucleation and initiation of micro-cracks. As the micro-crack grows, the size of the plastic zone at the crack tip gets larger, as well as the stress intensity factor. This would therefore result in a higher level of stress in a local region near the micro-cracks, as compared with neighboring regions. As a result, numerous micro-cracks were initiated, propagated, and then coalesced in Zone II. Namely, the formation of Zone II corresponds to a multi-crack propagation stage in the process of fatigue failure. This is the reason why there are a large number of radial ridges in Zone II, which make it relatively rough. Afterward, when the stress intensity reaches the crack propagation threshold value, namely, the $\Delta \mathrm{K}$ value of the edge of Zone II, the crack propagation pattern 
changes again. In some researches of high-strength steels, it was proposed that $[46,47]$ the $\Delta \mathrm{K}_{\mathrm{RA}}(\Delta \mathrm{K}$ Zone II in the present case) value corresponds to the relevant threshold for long crack growth. Therefore, the growth pattern of the crack in Zone III switched from the multiple short crack growth to the steady single long-crack growth that follows Paris law.

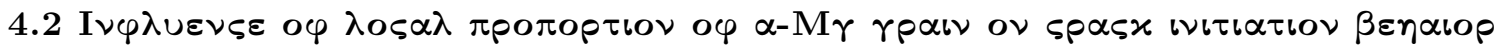

As a dual-phase Mg-Li alloy, there may also exist hard and soft phases in the LZ91 alloy, just similar to the duplex stainless steels that have been studied before. The plastic deformation will preferentially accumulate in the soft phase during the subsequent micro-crack initiation and early propagation [35, 36]. Therefore, we calculated the proportion of $\alpha-\mathrm{Mg}$ and $\beta$-Li phases in the region near the crack initiation sites. In this way, we tried to explore whether the difference in local microstructure will affect the fatigue performance of the material. As presented in Figure 10, we calculated the area of $\alpha-M g$ grains in the region near the crack initiation site in each fracture topography. Note that the area of each calculated region is the same, and the area of the calculated region is determined by the largest area of Zone I (specimen No. 5). In this way, the proportion of $\alpha-\mathrm{Mg}$ grains near the crack initiation site can be quantified more intuitively. The conversion of the area of $\alpha-\mathrm{Mg}$ and $\beta$-Li grains was done by the mathematical software. The statistical results of the area of $\alpha$ and $\beta$ phases in the calculated region are listed in Table $\mathbf{5}$, and the $\alpha$-Mg ratio-Fatigue life diagram is presented in Fig 10b, respectively. It is obvious that there is a simple linear relationship between the fatigue life of the material and the proportion of $\alpha-\mathrm{Mg}$ phase in the region near the crack initiation site. Namely, the lower the proportion of $\alpha-\mathrm{Mg}$ phase in the region is, the longer the fatigue life will be. Besides, it is worth noting that the proportion of $\alpha-\mathrm{Mg}$ phase in most specimens is below the average value of the material. Based on the discussion above, it can be preliminarily inferred that the $\alpha$ phase is a strengthening phase in the material as compared with the $\beta$ phase. In other words, fatigue crack is more likely to nucleate and grow in a local region, where the proportion of $\alpha-\mathrm{Mg}$ grains is relatively low. As demonstrated in Section 3.2 , however, the hardness and the modulus of $\alpha-\mathrm{Mg}$ and $\beta$-Li phase vary barely. As pointed above, the crystallographic structure of the two phases is different, in which the $\alpha-\mathrm{Mg}$ phase is the HCP, and the $\beta$-Li phase is the BCC structure, respectively. According to previous studies, the texture of magnesium alloy will significantly affect the fatigue behavior of the material. Therefore, in the present work, the most likely cause for this may be the enhancing effect of the texture and the grain orientation of $\alpha-\mathrm{Mg}$ grains.

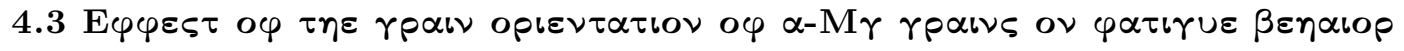

It is demonstrated in the previous section that there is a certain relationship between the local proportion of $\alpha-\mathrm{Mg}$ in the region near the crack initiation site and the fatigue life of the material. Since the hardness and elastic modulus of the two phases vary slightly, the microstructure of the material may be the governing affecting factor of the fatigue properties. As displayed in Figure 2, the grain orientation of most $\alpha-\mathrm{Mg}$ grains is around $\{10-10\}$ and $\{11-20\}$ cylindrical direction, namely, the basal plane of them is almost parallel to the loading direction during fatigue tests. It is well known that the magnesium possesses only 12 slip systems [48-50], with three basal slip systems $\{0001\}<-12-20>$, three prismatic slip systems $\{10-10\}<-12-20>$, and six pyramidal slip systems $\{10-10\}<11-23>$. It has been reported that the initial CRSS (critical resolved shear stress) value of basal slip systems (about $0.45^{\sim} 0.81 \mathrm{MPa}$ ) and $\{10-12\}$ tensile twinning deformation (about 2.0 2.8 MPa) is far below that of non-basal slip systems (about $39.2 \mathrm{MPa}$ of prismatic slip and $45^{\sim} 91$ $\mathrm{MPa}$ of pyramidal slip) and compressive twinning deformation (about 76-153 MPa) at room temperature. Thus, the basal slip systems and tensile twinning deformation are most easily activated during the process of deformation. However, due to the fact that the basal plane of the $\alpha$ phase is nearly perpendicular to the loading direction, the activation of basal slip is highly hindered. Meanwhile, the loading-depth curve of the $\alpha$ $\mathrm{Mg}$ grain obtained by the nano-indentation test demonstrates that the $\alpha$-Mg and $\beta$-Li phases exhibit distinct characteristics. Clearly, the loading-depth curve of the $\alpha-M g$ phase exhibits a "pop-in" effect comparing to the $\beta$-Li phase, which is proved to be caused by the twinning deformation of $\alpha$-Mg grains under compressive loading. Therefore, it can be preliminary deduced that the twinning deformation is the predominating deformation pattern of $\alpha-\mathrm{Mg}$ grains when subjected to axial loading.

As been revealed in Section 4.2, there is a linear relationship between the fatigue life and the proportion of $\alpha$ - 
$\mathrm{Mg}$ phase in the region near the crack initiation site. Namely, a higher local proportion of $\alpha$-Mg phase, which acts as a hard phase, seems to increase the fatigue life of the material to a certain extent. Two main reasons, which make the $\alpha-M g$ phase the hard phase in the material, can be concluded from the above discussion. Firstly, the slip system of the HCP structure is less than the BCC structure, thus the local coordinate deformability of the $\beta$-Li phase is much better than that of the $\alpha$-Mg phase. Secondly, the orientation of the $\alpha-\mathrm{Mg}$ phase is mainly around the $\{10-10\}$ and $\{11-20\}$ cylindrical direction, which makes the basal slip of the $\alpha-\mathrm{Mg}$ phase difficult to be activated. Namely, the $\alpha-\mathrm{Mg}$ phase becomes the hard phase in the material, whose deformability is much lower than the $\beta$-Li phase. As a result, the $\beta$-Li phase takes the major part of the plastic deformation during the fatigue testing, and the $\alpha-\mathrm{Mg}$ grains become a strengthening phase that is difficult to deform. Therefore, the fatigue crack tends to nucleate and initiate from the region where the $\alpha-\mathrm{Mg}$ phase is relatively sparse.

\subsection{A proposed model of the fatigue behavior of LZ91 dual-phase Mg-Li alloy}

As discussed above, small crack initiation and propagation are highly affected by the microstructure of the material. Figure 11 thus presents the schematic illustration of crack initiation and early propagation process. The stripe phase in white is the $\alpha$-Mg phase, and the matrix in blue is the $\beta$-Li phase, respectively. Severe plastic deformation tends to occur in the 'soft' phase, i.e., the $\beta$-Li phase during cyclic loading, as shown in Figure 11a. As crack grows and has to pass through $\alpha$-Mg grains, the crack propagation will be inevitably hindered by $\alpha-\mathrm{Mg}$ grain due to the hard grain orientations. As a result, there are some turning points (marked by black arrows) in the crack path when it passes through $\alpha$-Mg grains, which make it appear more tortuous. Eventually, crack continues to propagate, resulting in the final failure of the material.

In the present investigation, the experimental results and microstructural analysis of the crack initiation will fill the gap of our understanding of the VHCF behavior of the dual-phase Mg-Li alloy. More importantly, they would provide more valuable advice to improve the anti-fatigue ability of the $\mathrm{Mg}$ - $\mathrm{Li}$ alloys.

\section{Conclusions}

In the present paper, the VHCF behavior of a dual-phase Mg-Li alloy (LZ91) was studied. The underlying mechanisms of the crack initiation and early propagation have been fully investigated. The following conclusions can be drawn from the discussions:

1. The S-N curve of the LZ91 alloy decreases continuously without a fatigue limit. The fatigue strength is evaluated to $78 \mathrm{MPa}$ at $10^{9}$ cycles, and the fatigue ratio of the material is calculated to 0.46 , respectively.

2. The $\alpha$-Mg grains in the material exhibit strong textures due to the extrusion, in which the basal planes $\alpha-\mathrm{Mg}$ grains are almost parallel to the loading direction. The twinning deformation is the predominant pattern of $\alpha-\mathrm{Mg}$ grains since the basal slip is highly hindered during fatigue tests, making the $\alpha-\mathrm{Mg}$ the strengthening phase in the material.

3. The fractography of the material presents a noticeable microstructural sensitivity. The distinct morphologies of Zone I, II, and III resulted from the differences in the size of the plastic zone at the crack tip.

4. The fatigue crack initiation is significantly affected by the local microstructure of the material. The proportion of the $\alpha-\mathrm{Mg}$ phase of the region near the crack initiation region is lower than the average level. Cracks tend to nucleate and initiate from the local region with fewer $\alpha$-Mg grains, and the fatigue life is linearly positively correlated with the proportion of $\alpha-\mathrm{Mg}$ phase in the region.

\section{Acknowledgment}

The authors gratefully acknowledge the financial support from the National Natural Science Foundation of China (Nos. 12072212 and 11832007), the National Key Research and Development Program of China (No. 2018YFE0307104) and the Applied Basic Research Programs of Sichuan Province (No.21YYJC2743).

\section{Author Contribution Statement}


Xiangyu WANG : Data curation, Methodology, Software, Writing - original draft. Chao HE : Project administration, Methodology, Software, Writing -review editing. Xue LI : Resources, Writing - original draft. Yongjie LIU : Formal analysis, Supervision. Qingyuan Wang : Conceptualization, Funding acquisition. Lang Li : Investigation, Visualization.Hong Zhang : Investigation, Visualization. Chong Wang : Formal analysis, Supervision.

\section{References}

[1] Z. Yang, J.P. Li, J.X. Zhang, G.W. Lorimer, J. Robson, Review on research and development of magnesium alloys, Acta metallurgica sinica (English letters) 21(5) (2009) 313-328.

[2] B.L. Mordike, T. Ebert, Magnesium: properties - applications - potential, Materials Science and Engineering: A 302(1) (2001) 37-45.

[3] V. Kumar, R. Shekhar, R. Balasubramaniam, K. Balani, Microstructure evolution and texture development in thermomechanically processed $\mathrm{Mg}-\mathrm{Li}-\mathrm{Al}$ based alloys, Materials Science and Engineering: A 547 (2012) 38-50.

[4] Z. Wu, R. Ahmad, B. Yin, S. Sandlöbes, W.A. Curtin, Mechanistic origin and prediction of enhanced ductility in magnesium alloys, Science 359(6374) (2018) 447-452.

[5] K. Srivastava, J.A. El-Awady, Deformation of magnesium during c-axis compression at low temperatures, Acta Materialia 133 (2017) 282-292.

[6] M.R. Barnett, M.D. Nave, C.J. Bettles, Deformation microstructures and textures of some cold rolled Mg alloys, Materials Science and Engineering: A 386(1-2) (2004) 205-211.

[7] R.-z. Wu, Y.-d. Yan, G.-x. Wang, L.E. Murr, W. Han, Z.-w. Zhang, M.-l. Zhang, Recent progress in magnesium-lithium alloys, International Materials Reviews 60(2) (2015) 65-100.

[8] Z. Trojanová, Z. Drozd, S. Kúdela, Z. Száraz, P. Lukáč, Strengthening in Mg-Li matrix composites, Composites Science and Technology 67(9) (2007) 1965-1973.

[9] J. Hirsch, T. Al-Samman, Superior light metals by texture engineering: Optimized aluminum and magnesium alloys for automotive applications, Acta Materialia 61(3) (2013) 818-843.

[10] Y. Zou, L. Zhang, Y. Li, H. Wang, J. Liu, P.K. Liaw, H. Bei, Z. Zhang, Improvement of mechanical behaviors of a superlight $\mathrm{Mg}$-Li base alloy by duplex phases and fine precipitates, Journal of Alloys and Compounds 735 (2018) 2625-2633.

[11] H.-Y. Wu, J.-C. Yan, H.-H. Tsai, C.-H. Chiu, G.-Z. Zhou, C.-F. Lin, Tensile flow and strain-hardening behaviors of dual-phase Mg-Li-Zn alloy thin sheets, Materials Science and Engineering: A 527(27-28) (2010) 7197-7203.

[12] C. Bathias, P.C. Paris, Gigacycle Fatigue in Mechanical Practice. Dekker, (2005).

[13] Q.Y. Wang, J.Y. Berard, A. Dubarre, G. Baudry, S. Rathery, C. Bathias, Gigacycle fatigue of ferrous alloys, Fatigue \& Fracture of Engineering Materials \& Structures 22(8) (1999) 667-672.

[14] S. Stanzl-Tschegg, H. Mughrabi, B. Schoenbauer, Life time and cyclic slip of copper in the VHCF regime, International journal of fatigue 29(9-11) (2007) 2050-2059.

[15] M.D. Sangid, The physics of fatigue crack initiation, International journal of fatigue 57 (2013) 58-72.

[16] K. Yang, C. He, Q. Huang, Z.Y. Huang, C. Wang, Q. Wang, Y.J. Liu, B. Zhong, Very high cycle fatigue behaviors of a turbine engine blade alloy at various stress ratios, International Journal of Fatigue 99 (2017) $35-43$.

[17] Y. Hong, Z. Lei, C. Sun, A. Zhao, Propensities of crack interior initiation and early growth for veryhigh-cycle fatigue of high strength steels, International Journal of Fatigue 58 (2014) 144-151. 
[18] Y. Murakami, Metal fatigue: effects of small defects and nonmetallic inclusions, Academic Press2019.

[19] F. Yang, S.M. Yin, S.X. Li, Z.F. Zhang, Crack initiation mechanism of extruded AZ31 magnesium alloy in the very high cycle fatigue regime, Materials Science and Engineering: A 491(1-2) (2008) 131-136.

[20] K. Tokaji, M. Kamakura, Y. Ishiizumi, N. Hasegawa, Fatigue behaviour and fracture mechanism of a rolled AZ31 magnesium alloy, International Journal of Fatigue 26(11) (2004) 1217-1224.

[21] J. Pan, P. Fu, L. Peng, B. Hu, H. Zhang, A.A. Luo, Basal slip dominant fatigue damage behavior in a cast Mg-8Gd-3Y-Zr alloy, International Journal of Fatigue 118 (2019) 104-116.

[22] D. Hanwu, W. Limin, L. Ke, W. Lidong, J. Bin, P. Fusheng, Microstructure and deformation behaviors of two Mg-Li dual-phase alloys with an increasing tensile speed, Materials \& Design 90 (2016) 157-164.

[23] F. Guo, L. Liu, Y. Ma, L. Jiang, Y. Zhang, D. Zhang, F. Pan, Slip behavior and its effect on rolling texture development in a dual-phase Mg-Li alloy, Journal of Alloys and Compounds 813 (2020) 152117.

[24] M. Shahzad, L. Wagner, Influence of extrusion parameters on microstructure and texture developments, and their effects on mechanical properties of the magnesium alloy AZ80, Materials Science and Engineering: A 506(1-2) (2009) 141-147.

[25] J. Bohlen, S. Yi, D. Letzig, K.U. Kainer, Effect of rare earth elements on the microstructure and texture development in magnesium-manganese alloys during extrusion, Materials Science and Engineering: A 527(26) (2010) 7092-7098.

[26] S.B. Yi, H.G. Brokmeier, J. Bohlen, D. Letzig, K.U. Kainer, Neutron diffraction study on the texture development during extrusion of magnesium alloy AZ31, Physica B: Condensed Matter 350(1-3) (2004) E507-E509.

[27] V. Kumar, A. Gupta, D. Lahiri, K. Balani, Serrated yielding during nanoindentation of thermomechanically processed novel $\mathrm{Mg}-9 \mathrm{Li}-7 \mathrm{Al}-1 \mathrm{Sn}$ and $\mathrm{Mg}-9 \mathrm{Li}-5 \mathrm{Al}-3 \mathrm{Sn}-1 \mathrm{Zn}$ alloys, Journal of Physics D: Applied Physics 46(14) (2013) 145304.

[28] W. Wang, C.B. Jiang, K. Lu, Deformation behavior of Ni3Al single crystals during nanoindentation, Acta materialia 51(20) (2003) 6169-6180.

[29] V. Kumar, K. Philippe, R. Shekhar, K. Balani, Processing and Nano-mechanical Characterization of Mg-Li-Al Based Alloys, Procedia Materials Science 5 (2014) 585-591.

[30] T. Guo, F. Siska, J. Cheng, M. Barnett, Initiation of basal slip and tensile twinning in magnesium alloys during nanoindentation, Journal of Alloys and Compounds 731 (2018) 620-630.

[31] X. Peng, X. Liang, W. Liu, G. Wu, H. Ji, X. Tong, L. Zhang, W. Ding, High-cycle fatigue behavior of Mg-8Li-3Al-2Zn-0.5 Y alloy under different states, Journal of Magnesium and Alloys (2020).

[32] C. He, Y. Liu, J. Li, K. Yang, Q. Wang, Q. Chen, Very-high-cycle fatigue crack initiation and propagation behaviours of magnesium alloy ZK60, Materials Science and Technology 34(6) (2018) 639-647.

[33] H. Liu, H. Wang, Z. Huang, Q. Wang, Q. Chen, Comparative study of very high cycle tensile and torsional fatigue in TC17 titanium alloy, International Journal of Fatigue 139 (2020).

[34] F. Liu, C. He, Y. Chen, H. Zhang, Q. Wang, Y. Liu, Effects of defects on tensile and fatigue behaviors of selective laser melted titanium alloy in very high cycle regime, International Journal of Fatigue 140 (2020).

[35] M.W. Tofique, J. Bergström, K. Svensson, S. Johansson, R.L. Peng, ECCI/EBSD and TEM analysis of plastic fatigue damage accumulation responsible for fatigue crack initiation and propagation in VHCF of duplex stainless steels, International Journal of Fatigue 100 (2017) 251-262.

[36] I.H. Lo, W.-T. Tsai, Effect of selective dissolution on fatigue crack initiation in 2205 duplex stainless steel, Corrosion Science 49(4) (2007) 1847-1861. 
[37] R. Strubbia, S. Hereñú, I. Alvarez-Armas, U. Krupp, Short fatigue cracks nucleation and growth in lean duplex stainless steel LDX 2101, Materials Science and Engineering: A 615 (2014) 169-174.

[38] J.K. Sahu, U. Krupp, H.J. Christ, Fatigue crack initiation behavior in embrittled austenitic-ferritic stainless steel, International Journal of Fatigue 45 (2012) 8-14.

[39] Y. Murakami, S. Kodama, S. Konuma, Quantitative evaluation of effects of non-metallic inclusions on fatigue strength of high strength steels. I: Basic fatigue mechanism and evaluation of correlation between the fatigue fracture stress and the size and location of non-metallic inclusions, International Journal of Fatigue 11(5) (1989) 291-298.

[40] C. He, Y. Liu, J. Li, K. Yang, Q. Wang, Q. Chen, Very-high-cycle fatigue crack initiation and propagation behaviours of magnesium alloy ZK60, Materials Science and Technology 34(6) (2017) 639-647.

[41] X.H. Shao, H.Q. Liu, H.J. Yang, C. He, N. Su, Y.J. Wu, Q. Chen, X.L. Ma, Enhanced very high cycle fatigue resistance of solution treated $\mathrm{Mg}-10 \mathrm{Gd}-3 \mathrm{Y}-1 \mathrm{Zn}-0.5 \mathrm{Zr}$ magnesium alloy containing long-period stacking ordered phase, Materialia 11 (2020).

[42] U. Karr, B.M. Schönbauer, H. Mayer, Near-threshold fatigue crack growth properties of wrought magnesium alloy AZ61 in ambient air, dry air, and vacuum, Fatigue \& Fracture of Engineering Materials \& Structures 41(9) (2018) 1938-1947.

[43] X. Pan, H. Su, C. Sun, Y. Hong, The behavior of crack initiation and early growth in high-cycle and very-high-cycle fatigue regimes for a titanium alloy, International Journal of Fatigue 115 (2018) 67-78.

[44] W. Li, H. Zhao, A. Nehila, Z. Zhang, T. Sakai, Very high cycle fatigue of TC4 titanium alloy under variable stress ratio: Failure mechanism and life prediction, International Journal of Fatigue 104 (2017) 342-354.

[45] K. Yang, Q. Huang, Q. Wang, Q. Chen, Competing crack initiation behaviors of a laser additively manufactured nickel-based superalloy in high and very high cycle fatigue regimes, International Journal of Fatigue 136 (2020).

[46] X. Pan, Y. Hong, High-cycle and very-high-cycle fatigue behaviour of a titanium alloy with equiaxed microstructure under different mean stresses, Fatigue \& Fracture of Engineering Materials \& Structures 42(9) (2019) 1950-1964.

[47] Y. Hong, C. Sun, The nature and the mechanism of crack initiation and early growth for very-high-cycle fatigue of metallic materials-An overview, Theoretical and Applied Fracture Mechanics 92 (2017) 331-350.

[48] W.B. Hutchinson, M.R. Barnett, Effective values of critical resolved shear stress for slip in polycrystalline magnesium and other hcp metals, Scripta Materialia 63(7) (2010) 737-740.

[49] T. Hama, H. Takuda, Crystal-plasticity finite-element analysis of inelastic behavior during unloading in a magnesium alloy sheet, International Journal of Plasticity 27(7) (2011) 1072-1092.

[50] T. Hama, N. Kitamura, H. Takuda, Effect of twinning and detwinning on inelastic behavior during unloading in a magnesium alloy sheet, Materials Science and Engineering: A 583 (2013) 232-241.

\section{Hosted file}

Figures-0420.pdf available at https://authorea.com/users/410198/articles/519671-crackinitiation-and-propagation-mechanisms-of-an-extruded-dual-phase-magnesium-lithium-alloyin-very-high-cycle-fatigue-regime

\section{Hosted file}

Table-0422.pdf available at https://authorea.com/users/410198/articles/519671-crackinitiation-and-propagation-mechanisms-of-an-extruded-dual-phase-magnesium-lithium-alloyin-very-high-cycle-fatigue-regime 